\title{
Study of corrosion-active microorganisms of the coastal zone of the Baltic and Barents seas
}

\author{
Galina Nianikova ${ }^{1, *}$, Andrey Bryukhanov ${ }^{2}$, Dmitry Vlasov $^{3}$, Margarita Mayorova $^{4}$, \\ Magomed Nurmagomedov ${ }^{5}$, Dmitry Akhaev $^{2}$, and Inga Tsarovtseva ${ }^{4}$ \\ ${ }^{1}$ Saint-Petersburg State Institute of Technology, Department Technology of Microbiological \\ Synthesis, Moskovsky prospect, 26, St. Petersburg, 190013, Russia \\ ${ }^{2}$ Lomonosov Moscow State University, Faculty of Biology, GSP-1, Leninskie Gory, 119991 Moscow, \\ Russia \\ ${ }^{3}$ Saint Petersburg State University, Faculty of Biology, 11 Lieutenant Shmidt Emb., St Petersburg \\ 199034, St. Petersburg, Russia \\ ${ }^{4}$ JSC "Vedeneev VNIIG", 21, Gzhatskaya str., Saint-Petersburg, 195220, Russia \\ ${ }^{5}$ JSC “Leningradskaya GAES”, Tsesarskogo str., 2, Murmashi, Murmansk, Russia
}

\begin{abstract}
The composition of microbial communities in coastal zones in the area of Kislogubskaya tidal power plant (the Barents Sea) and near Saint Petersburg Flood Prevention Facility Complex in the Gulf of Finland (Baltic Sea) was studied. The enrichment and pure cultures of microorganisms of various groups (sulfate-reducing, sulfur-oxidizing, nitrifying, ammonifying and iron-oxidizing bacteria) have been isolated, their cultural and physiological-biochemical properties, as well as their ability to induce corrosion of different materials used in the construction of hydraulic structures, have been studied.
\end{abstract}

\section{Introduction}

For hydraulic structures located in different climatic zones, on the sea coasts, as well as in conditions of long-term operation (more than 50 years), their reliability and durability are of great importance. Currently, class of problems related to corrosion induced damages of concrete and reinforced concrete structures has expanded, which can contribute to a more rapid degradation of hydraulic structures.

In the design and operation of hydraulic structures, as well as to assess their service life, it is necessary to take into account many factors, including the nature and rates of corrosion processes. Corrosion destruction of structural materials operating in a maritime climate is different depending on their location: a zone of atmospheric influence, a zone of variable sea water level, etc. [1]. Along with the study of physical and chemical corrosion processes, it is of great interest to study the biological corrosion of structural materials located in different climatic zones. At the same time, there is a number of general stages of the colonization of materials by microbial communities. The first stage is associated with the transfer of microorganisms to the surface of various materials and metal structures [2]. This

* Corresponding author: galanyan@mail.ru 
is followed by the adsorption of microorganisms on the surfaces of structures with the subsequent formation of microcolonies and biofilms [3,4]. This process is accompanied by the release into the external environment of corrosive products of microbial metabolism: acids (organic and inorganic), exopolysaccharides, hydrogen sulfide, various enzymes, etc. The accumulation of these metabolites is an important factor in the biochemical degradation of materials [5].

Microorganisms cause biocorrosion processes in wide ranges of temperatures, humidity, $\mathrm{pH}$, salinity, etc. The participation in the corrosion process of microorganisms of various physiological groups and their possible associations, as well as their high adaptability to various habitat conditions and nutrient sources, complicates the development of universal methods of protecting structures from biological damages.

The purpose of this study is to assess the diversity, to isolate pure cultures and to study the impact of microorganisms and microbial associations of the coastal zone of the northern seas on the structural and physical-mechanical properties of different materials used in hydraulic engineering.

\section{Materials and methods}

In the area of the Kislogubskaya tidal power plant (TPP) of the branch of JSC "Leningradskaya GAES" (hydroelectric power station), located in the north of the Kola Peninsula in the Kislaya Guba of the Barents Sea (Murmansk Oblast), and at Saint Petersburg Flood Prevention Facility Complex (FPFC) in the Gulf of Finland (the Baltic Sea), from 2018 to 2020 samples of various construction materials were exhibited, namely cubes made of concrete mix, cubes made of wood (pine), reinforcement with application of an organosilicate protective coating and without application (Fig. 1).

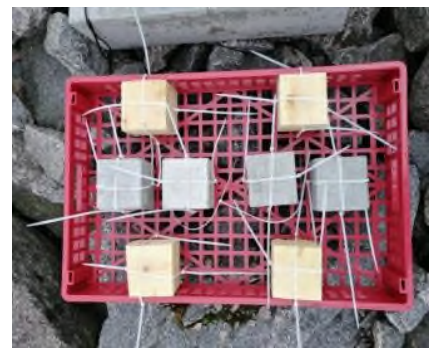

a

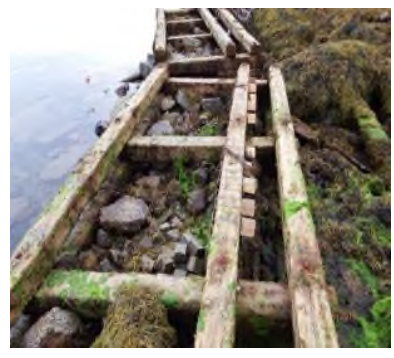

b

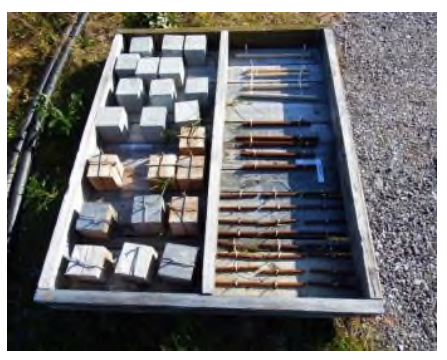

$\mathrm{c}$

Fig. 1. Testing benches for full-scale field tests of structural materials: a) an example of samples exhibited in the Gulf of Finland of the Baltic Sea in the splash zone; b) littoral testing bench with samples exhibited in the area of the Kislogubskaya TPP (the Barents Sea); c) atmospheric testing bench with samples exhibited in the area of the Kislogubskaya TPP (the Barents Sea).

For microbiological studies, samples were taken from the surface of the exposed materials, as well as samples of water and bottom sediments in the coastal zone of the Barents Sea in the area of the Kislogubskaya TPP and in the coastal zone of the Baltic Sea near the FPFC. The identification and study of microorganisms that are potentially corrosive were carried out by classical microbiological and molecular genetic methods. To isolate different groups of aerobic microorganisms into enrichement cultures the following selective nutrient media were used:

for nitrifying bacteria, Winogradsky's medium, g/l: (NH4)2SO4 - 2.0; K2HPO4 x $12 \mathrm{H} 2 \mathrm{O}$ - 1.0; $\mathrm{MgSO} 4$ - 0.5; FeSO4 × 7H2O - 0.01; $\mathrm{NaCl}-2.0 ; \mathrm{CaCO} 3$ - 1.0; $\mathrm{pH}$ - 7.5; 
for sulfur-oxidizing (thionic) bacteria, Beijerinck's medium, g/l: Na2S2O3 $\times 5 \mathrm{H} 2 \mathrm{O}-$ $5.0 ; \mathrm{NH} 4 \mathrm{Cl}-0.1 ; \mathrm{NaHCO} 3-1.0 ; \mathrm{Na} 2 \mathrm{HPO} 4 \times 12 \mathrm{H} 2 \mathrm{O}-0.2 ; \mathrm{FeSO} 4 \times 7 \mathrm{H} 2 \mathrm{O}-0.01 ; \mathrm{pH}-$ 9.2;

for iron-oxidizing bacteria, Zakharova's medium, g/l: (NH4)2SO4 - 0.5; NaNO3 - 0.5; $\mathrm{K} 2 \mathrm{HPO} 4-0.5$; MgSO4 x 7H2O - 0.5; citric acid - 10.0; sucrose - 2.0; tryptone - 1.0; $\mathrm{FeSO} 4 \times 7 \mathrm{H} 2 \mathrm{O}-5.9 ; \mathrm{pH}=6.8$;

for ammonifying bacteria, $\mathrm{g} / \mathrm{l}$ : fish meal hydrolyzate $-1.0 ; \mathrm{NaCl}-0.5 ; \mathrm{pH}-6.8$;

the total microbial count of aerobic organotrophic bacteria was determined on the GMF medium (enzymatic meat hydrolyzate);

detection of micromyces was carried out on a Czapek's medium, g/l: NaNO3 -2.0; K2HPO4 - 1.0; MgSO4 - 0.5; KCl - 0.5; FeSO4 - traces; $\mathrm{pH}$ - 6.8-7.0.

In culture studies, both liquid and solid agar (2\% agar) nutrient media were used.

The cultivation was carried out at a temperature of $28-30{ }^{\circ} \mathrm{C}$ for $3-7$ days in Petri dishes and in submerged conditions in Erlenmeyer flasks at a stirring mode of $220 \mathrm{rpm}$. Microscopy after staining of cells with fuchsine was performed using a light microscope at magnifications of $400 \times$ and $900 \times$.

Classical microbiological methods were used to determine the cultural, morphological, physiological and biochemical characteristics of the isolated aerobic cultures. The morphological description of colonies of microorganisms on the surface of agar nutrient media was carried out by visual inspection. Behavior towards oxygen was determined by the method of injecting into the columns of the appropriate agar nutrient medium. To determine the temperature optimum, the cultivation was carried out on solid and liquid nutrient media in various temperature ranges $\left(+4{ }^{\circ} \mathrm{C},+28{ }^{\circ} \mathrm{C}\right.$, and $\left.+45^{\circ} \mathrm{C}\right)$. The growth of cultures in nutrient media with different $\mathrm{pH}$ ranges was also studied.

To obtain enrichment cultures of anaerobic sulfate-reducing bacteria (SRB), $1 \mathrm{~cm} 3$ of bottom sediment was introduced into Hungate tubes with Widdel's sterile liquid nutrient medium for marine forms of SRB with the addition of lactate $(10 \mathrm{mM})$ and yeast extract $(0.5 \mathrm{~g} / \mathrm{L})$ or acetate $(10 \mathrm{mM})$ and yeast extract $(0.5 \mathrm{~g} / \mathrm{l})$ as electron donors. Composition of Widdel's nutrient medium for marine forms of SRB [6], g/l: Na2SO4 - 4.0; KH2PO4 0.2; $\mathrm{NH} 4 \mathrm{Cl}-0.25 ; \mathrm{NaCl}-13.5 ; \mathrm{MgCl} 2 \times 6 \mathrm{H} 2 \mathrm{O}-0.4 ; \mathrm{KCl}-0.5 ; \mathrm{CaCl} 2-0.1$; supplements to the mineral salts, $\mathrm{ml} / \mathrm{l}$ : vitamins according to Widdel - 1.0; trace elements solution SL-10 $1.0 ; \mathrm{NaHCO} 3$ (10\% solution) $-10.0 ; \mathrm{Na} 2 \mathrm{~S}$ ( $5 \%$ solution) $-10.0 ; \mathrm{FeSO} 4 \times 7 \mathrm{H} 2 \mathrm{O}(5 \%$ solution) -0.5 .

Inoculations of anaerobic SRB cultures were carried out using sterile syringes; the inoculum volume was $10 \%$. The cultivation was carried out at a temperature of $20-25{ }^{\circ} \mathrm{C}$ in the dark for 5-25 days. Growth control was performed using light microscopy. For pure SRB cultures, their growth was also controlled by determining hydrogen sulfide by the colorimetric method with N,N-dimethyl-p-phenylenediamine.

Isolation of pure SRB cultures from active enrichment cultures was carried out by the method of limiting dilutions. Ten Hungate anaerobic tubes with a sterile liquid Widdel's nutrient medium for marine forms of SRB with a volume of $9 \mathrm{ml}$ were used. With a sterile syringe, $1 \mathrm{ml}$ of the grown enrichment culture was taken and transferred sequentially into tubes with a nutrient medium in the dilution range from 10-1 to 10-10. After incubation of the diluted cultures for 10 days a microscopy was carried out. If no more than three or four morphological types of cells were seen in the corresponding variant, inoculation was carried out in the column of the corresponding agar nutrient medium to obtain individual colonies.

For taxonomic identification, total DNA was isolated from bacterial biomass using the Genomic DNA Purification Kit (Thermo Fisher Scientific, USA), following the manufacturer's instructions. The concentration of the obtained DNA samples was 30-50 $\mu \mathrm{g} / \mathrm{ml}$. Sequencing of PCR fragments of the 16S rRNA gene amplified using $11 \mathrm{f}$ and $1492 \mathrm{r}$ 
primers [7] was performed according to Sanger's method using the BigDye Terminator v. 3.1 (Applied Biosystems, USA) on an ABI PRIZM 3730 genomic analyzer (Applied Biosystems, USA) according to the manufacturer's instructions. The primary analysis of the similarity of the 16S rRNA gene nucleotide sequences of the studied strains was carried out using BLASTN and RDP Classifier software packages.

To study the processes of biocorrosion of structural materials in laboratory conditions, an appropriate agar nutrient medium was poured into sterile plastic dishes $24 \times 24 \mathrm{~cm}$ in size, aerobic bacterial cultures were inoculated, and plates of structural steel St 20 pretreated with a disinfectant solution were placed on the surface of the inoculated medium. The plates were exposed at room temperature in the dark for 1 year. The manifestations of biocorrosion were assessed visually, as well as using a digital microscope, which makes it possible to photograph samples with signs of biocorrosion. In addition, samples of structural steel were exposed in the form of small pieces of reinforcement in Hungate tubes with anaerobic cultures of sulfate-reducing bacteria.

The influence of exposure conditions and the development of biofouling on the strength characteristics of concrete were evaluated by checking concrete blocks exposed on climatic testing bench for compressive strength in accordance with GOST 10180-2012 "Concretes. Methods for strength determination using reference specimens", determination of density (bulk density) of concrete samples was carried out in accordance with GOST 12730.1-78 "Concretes. Methods of determination of density".

\section{Results and discussion}

The analyzed samples taken from the testing benches in the area of the Kislogubskaya TPP (the Barents Sea) were characterized by different degrees of biofouling. A visual inspection of the exposed samples demonstrated that the samples exposed in the littoral zone were biofouled most significantly. The development of microbial biofilms was recorded on the surface of samples taken from the atmospheric testing bench. The nature and composition of microbial biofilms, as well as the degree of biofouling, depended to a great extent on the nature of the material surface and its physicochemical properties.

Thus, the surface of concrete after exposure of the samples on an atmospheric testing bench was characterized by the initial development of the structures of filamentous fungi and demonstrated signs of weak surface destruction of the material. Mycological analysis of these samples showed the dominance of fungi of the genus Cladosporium on the surface of concrete samples.

The concrete surface after exposure of the samples at the littoral testing bench in the area of the Kislogubskaya TPP was distinguished by more intensive colonization by microorganisms (growth of filamentous structures of cyanobacteria and microalgae, the formation of primary biofilms) in comparison with the samples from the atmospheric testing bench.

As a result of incubation of aerobic cultures of microorganisms, it was noted that on the 3rd day the growth of thionic and nitrifying bacteria was absent, and on the 7 th day it manifested itself in all samples taken from the reinforcement steel exposed in the atmospheric zone, and in most probes taken from the samples exposed in littoral zone. The highest growth on all nutrient media was recorded in samples taken from reinforcement of various diameters (except for reinforcement with a protective coating), as well as from samples of wood with or without a protective coating. Ammonifying bacteria were found in all samples tested. The total microbial count of heterotrophic bacteria on GMF medium reached $106 \mathrm{CFU} / \mathrm{ml}$ washout from the surface of the material sample. At the same time, the highest bacterial content was noted in testing probes taken from the samples of reinforcement without a protective coating, wood without a protective coating, and 
concrete. The presence of micromycetes was most noticeable in testing probes from samples of concrete, reinforcement, and wood without a protective coating, taken at an atmospheric testing bench. Anaerobic sulfate-reducing bacteria developed actively on reinforcement samples.

The morphology of colonies of all isolated aerobic cultures was studied. On the GMF medium, mucous, opaque, spreading over the medium, milky and cream-colored colonies predominated, reaching $8 \mathrm{~mm}$ in diameter. Along with them, yellow-orange numerous small colonies (up to $3 \mathrm{~mm}$ in diameter) developed in a great number. Abundant mucus production was characteristic of bacterial colonies in most cases. It is interesting to note that the same colonies developed on Czapek's nutrient medium, which was used to isolate fungi. This fact indicates the possibility of coexistence of fungi and organotrophic bacteria (bacterial-fungal associations), which have rather similar trophic needs. On Czapek's nutrient medium supplemented with calcium, a well-pronounced formation of acids by organotrophic bacteria and micromycetes was observed, which is one of the key factors of microbial corrosion. Acid-forming microorganisms were noted on all the studied substrates, however, their greatest development, like sulfate-reducing bacteria ones, was obtained in variants with corrosive reinforcement.

The primary analysis of the similarity of the 16S rRNA gene nucleotide sequences of the studied microorganisms was carried out using the BLASTN and RDP Classifier software packages. According to the results of BLAST analysis, isolated from a sample of reinforcement placed on a testing bench in the atmospheric zone of the Kislogubskaya TPP, the pure culture turned out to be the closest to the type strain Pseudomonas fluorescens CCM 2115, the level of nucleotide sequence similarity was $99.80 \%$.

Isolation of SRB pure cultures from active enrichment cultures, which represented cells in the form of vibrios and short rods with a small amount of spirilla, was performed using the method of limiting dilutions. After incubation of diluted up to 10-10 cultures for 10 days, a microscopy was carried out. For inoculation into the column of the agar nutrient medium, Widdel's liquid and agar media ( $2 \%$ of agar) for marine forms of SRB were used. After 2-4 weeks of incubation at room temperature, tubes were selected in which individual colonies could be clearly distinguished in agar nutrient medium. Then, the tubes were broken in a laminar flow hood, individual colonies were sterilely removed from the agar columns with a scalpel, and each of them was transferred into Hungate anaerobic tubes with a sterile Widdel's liquid medium for marine forms of SRB. Then, using a vacuum degassing unit with sterile membrane bacterial filters, oxygen in the medium, which got into the test tubes during inoculation of each colony, was removed, and they were blown off with sterile argon [8].

After 1-2 weeks, the purity of the cultures was assessed using light microscopy. To date, it has been possible to isolate two pure cultures of SRB from the bottom sediments of the Gulf of Finland of the Baltic Sea (Fig. 2a) and one pure culture of SRB from the bottom sediments in the area of the Kislaya Guba of the Barents Sea (Fig. 2b). All obtained SRB pure cultures grew well and formed hydrogen sulfide at room temperature $\left(23-25{ }^{\circ} \mathrm{C}\right)$ and $\mathrm{pH} 7.5$, reaching the maximum biomass yield in 24-48 $\mathrm{h}$ when growing on Widdel's nutrient medium for marine forms of SRB with lactate. On a medium with acetate, obtained SRB pure cultures developed much more slowly.

BLAST analysis of a $1495 \mathrm{bp}$ fragment of the 16S rRNA gene nucleotide sequence revealed $98.8 \%$ similarity of the pure culture isolated from the bottom sediment in the area of the Kislaya Guba of the Barents Sea near the Kislogubskaya TPP to the gram-negative halophilic sulfate-reducing bacterium Halodesulfovibrio aestuarii NCIMB 9335 [9].

According to the results of BLAST analysis of the 16S rRNA gene fragments with a length of 1486-1495 bp for two pure cultures isolated in the area of the S-1 ship pass in the Gulf of Finland of the Baltic Sea, they turned out to be the closest to Desulfovibrio 
oxamicus DSM 1925 (99.33\% similarity) and Desulfovibrio vulgaris Hildenborough (99.06\% similarity) respectively.

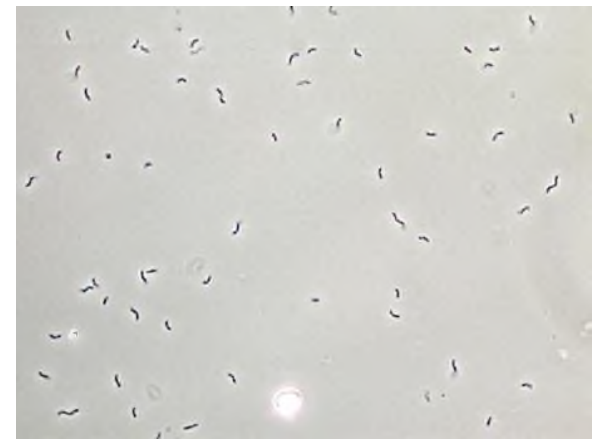

a

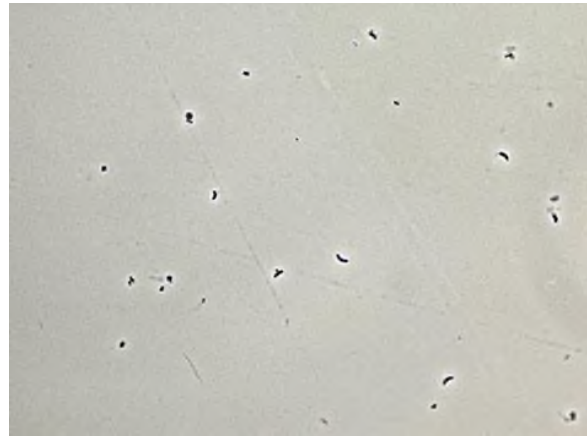

b

Fig. 2. Pure SRB culture of the genus Desulfovibrio isolated from the bottom sediment in the area of S-1 ship pass in the Gulf of Finland of the Baltic Sea (a). Pure SRB culture of the genus Halodesulfovibrio isolated from the bottom sediment in the area of the Kislogubskaya TPP, the Barents Sea (b). Light microscopy (400×).

In laboratory conditions, model experiments were carried out in order to study the processes of biocorrosion of the investigated structural materials. For this, samples of St20 structural steel were tested, which were inoculated with enrichment cultures of thionic bacteria of the genera Thiosphaera and Thiobacillus, nitrifying bacteria belonged to the genera Nitrobacter, Nitrococcus and Nitrosomonas, and iron-oxidizing bacteria of the genus Bacillus.

The first signs of corrosion on steel plates were noted as early as a week after the start of the experiment in the variant with thionic bacteria. Three weeks later, a thin mucous zone painted in a rusty brown color formed along the border of the plates and the nutrient medium with bacteria, and signs of corrosion appeared on the plates themselves. After 2 months, the rusty mucous zone around the plates reached $1 \mathrm{~cm}$, and the formation of growths was noted on the corrosive plates themselves (Fig. 3a). After 5 months of the experiment, the growths rose above the plate to a height of more than $1 \mathrm{~cm}$ and had a characteristic mushroom-like shape (Fig. 3b).

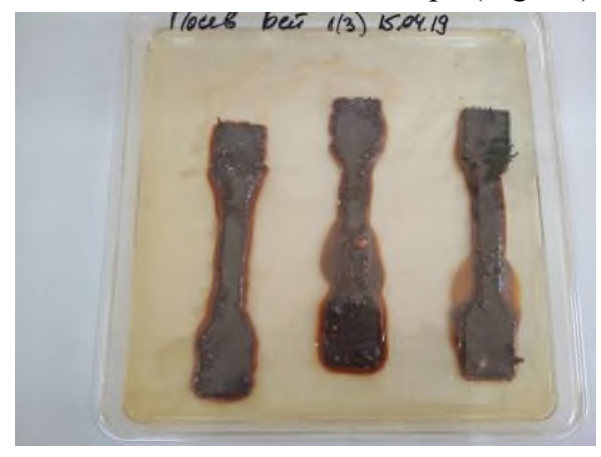

a

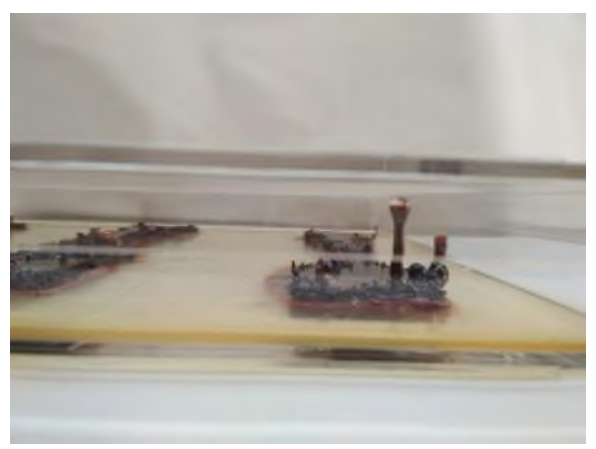

b

Fig. 3. Plates of St-20 structural steel in the presence of thionic bacteria of the genus Thiobacillus (a 60 days after inoculation, $\mathrm{b}-5$ months after inoculation).

In this case, a shiny mucous zone was formed along the entire length of the steel plates. The corrosive process developed further throughout the year, which was accompanied by 
an increase in the diameter of the drip zone around the plates and an increase in the number and size of new formations (growths) forming on corrosive steel plates.

Enrichment cultures of thionic (sulfur-oxidizing) bacteria exhibited the highest corrosive activity with respect to samples made of St-20 structural steel. The corrosion rate under the influence of these bacteria was the highest. Under the influence of heterotrophic iron-oxidizing bacteria, the corrosion process became significant only six months after the beginning of the experiment. Nitrifying bacteria did not show corrosive activity towards modelling plates.

The first signs of corrosion on the steel reinforcement were noted as early as 3 days after the start of the experiment in the variant with anaerobic sulfate-reducing bacteria. A week later, corrosion damage to the reinforcement and a dense black deposit of iron sulfide were already noticeable (Fig. 4).

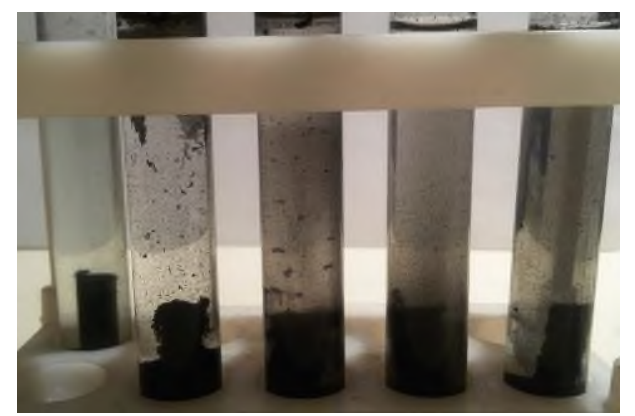

Fig. 4. Samples of steel reinforcement in the presence of sulfate-reducing bacteria of the genus Desulfovibrio three weeks after inoculation. At the left - the control tube with uninoculated nutrient medium.

Data on the influence of specimen exposure conditions on the strength characteristics of concrete were obtained during the study of concrete samples for compressive strength. The results of physical and mechanical studies of concrete samples are presented in table 1 .

Table 1. Results of physical and mechanical studies of concrete samples.

\begin{tabular}{|c|c|c|c|c|}
\hline \multirow{2}{*}{$\begin{array}{c}\text { Date of } \\
\text { removal of } \\
\text { samples }\end{array}$} & \multicolumn{4}{|c|}{ Concrete strength in a series of samples, MPa } \\
\cline { 2 - 5 } & $\begin{array}{c}\text { Control } \\
\text { samples }\end{array}$ & $\begin{array}{c}\text { Kislogubskaya TPP } \\
\text { (the Barents Sea), } \\
\text { littoral testing }\end{array}$ & $\begin{array}{c}\text { Kislogubskaya TPP } \\
\text { (the Barents Sea), } \\
\text { atmospheric testing }\end{array}$ & $\begin{array}{c}\text { Gulf of } \\
\text { Finland (the } \\
\text { Baltic Sea), } \\
\text { splash zone }\end{array}$ \\
\hline 05.09 .2019 & 54,34 & 47,50 & 50,15 & - \\
\hline 30.04 .2020 & 49,40 & 26,25 & 54,05 & 49,70 \\
\hline
\end{tabular}

The data obtained from physical and mechanical studies of concrete samples demonstrate that the strength of concrete at the littoral testing bench in the area of the Kislogubskaya TPP in relation to the control sample has decreased almost twice, while at the atmospheric testing bench it almost did not change.

\section{Conclusion}


The studies carried out made it possible to identify corrosive microorganisms of various physiological groups in samples taken from the surface of exposed structural materials, as well as in water and bottom sediment samples taken in the Gulf of Finland of the Baltic Sea near the Flood Prevention Facility Complex (St. Petersburg), and near the Kislogubskaya TPP in the southern part of the Barents Sea. Among the corrosive microorganisms identified in the course of the work, it is necessary to highlight sulfate-reducing, sulfuroxidizing, nitrifying and iron-oxidizing bacteria. Laboratory studies have shown that enrichment cultures of thionic (sulfur-oxidizing) bacteria, as well as sulfate-reducing bacteria, showed the greatest corrosive activity towards samples of structural steels.

A pure bacterial culture was identified, isolated from the surface of the reinforcement sample, which was exhibited at the atmospheric testing bench at the Kislogubskaya TPP. According to the results of BLAST analysis, this pure culture turned out to be the closest to the type strain Pseudomonas fluorescens CCM 2115 (the similarity level for the 16S rRNA gene is $99.80 \%$ ).

Also, pure cultures of anaerobic sulfate-reducing bacteria were successfully isolated from the bottom sediment samples taken in the Kislaya Guba of the Barents Sea near the Kislogubskaya TPP and in the area of the S-1 ship pass in the Gulf of Finland of the Baltic Sea. BLAST analysis of the $16 \mathrm{~S}$ rRNA gene fragments revealed $98.8 \%$ similarity of the pure culture isolated from the bottom sediment at the Kislogubskaya TPP to the halophilic sulfate-reducing bacterium Halodesulfovibrio aestuarii NCIMB 9335. According to the results of BLAST analysis of the 16S rRNA gene fragments for two cultures, isolated from the bottom sediments in the area of the S-1 shippass in the Gulf of Finland of the Baltic Sea, they turned out to be the closest to Desulfovibrio oxamicus DSM 1925 (99.33\% similarity) and Desulfovibrio vulgaris Hildenborough (99.06\% similarity).

It should be noted that the constant presence of potentially corrosive groups of microorganisms [10-12] in the sea water column and bottom sediments indicates the possibility of the formation of aggressive microbial communities in the form of biofouling and biofilms on hydraulic structures, which can significantly affect the operation of these structures and requires the development of protective measures against biofouling and biological damage.

The study was supported by the RFBR grant 18-29-05031 mk.

\section{References}

1. R.A. Markovich, Hydrotechnics 4, 56-59 (2009)

2. V.I. Solomatov, V.T. Erofeev, V.F. Smirnov, Biological resistance of materials (Saransk: Publishing house Mord. University, 2001)

3. L. Procópio, World J Microbiol Biotechnol. 35 (5) (2019)

4. A. Vigneron, E.B. Alsop, B. Chambers, B.P. Lomans, I.M. Head, N. Tsesmetzis. Appl Environ Microbiol 82 (8), 2545-2554 (2016)

5. A.L. Bryukhanov, D.Yu. Vlasov, M.A. Mayorova, I.M. Tsarovtseva, Hydraulic engineering 7, 7-13 (2020)

6. F. Widde, F. Bak. The Prokaryotes, $2^{\text {nd }}$ Edition (Ed. by A. Balows, H.G. Trüper, M. Dworkin, W. Harder, and K.-H. Schleifer), NY: Springer-Verlag. 4, 3352-3378 (1992)

7. D. J. Lane, In: Nucleic Acid Techniques in Bacterial Systematics (ed. By E. Stackebrandt, M. Goodfellow). Chichester: John Wiley \& Sons, Ltd. 115-175 (1991) 
8. A.L. Bryukhanov, A.I. Netrusov, A.I. Shestakov, I.B. Kotova. Methods of study of anaerobic microorganisms. The practical manual (Moscow, Scientific Library of Lomonosov Moscow State University, 2015)

9. Y. Shivani, Y. Subhash, C. Sasikala, C.V.Ramana, Int. J. Syst. Evol. Microbiol. 67(1), 87-93 (2017)

10. V. Moura, I. Ribeiro, P. Moriggi, A. Capao, C. Salles, S. Bitati, L. Procopio, Arch. Microbiol. 200(10), 1447-1456 (2018)

11. H. Makita, World J. Microbiol. Biotechnol. 34(8), 110 (2018)

12. D. Enning, H. Venzlaf, J. Garrelfs, H.T. Dinh, V. Meyer, K. Mayrhofer, A.W. Hassel, M. Stratmann, F. Widdel, Environ. Microbiol. 14(7), 1772-1787 (2012) 